\title{
Evidence of impaired neuromuscular responses in the support leg to a destabilizing swing phase perturbation in hemiparetic gait
}

\author{
Bahar Sharafi ${ }^{1,2,3}$ • Gilles Hoffmann ${ }^{2,3}$ • Andrew Q. Tan ${ }^{3,4}$ - Yasin Y. Dhaher $2,3,4,5$
}

Received: 4 August 2015 / Accepted: 26 July 2016 / Published online: 5 August 2016

(C) The Author(s) 2016. This article is published with open access at Springerlink.com

\begin{abstract}
The neuromuscular mechanisms that underlie post-stroke impairment in reactive balance control during gait are not fully understood. Previous research has described altered muscle activations in the paretic leg in response to postural perturbations from static positions. Additionally, attenuation of interlimb reflexes after stroke has been reported. Our goal was to characterize post-stroke changes to neuromuscular responses in the stance leg following a swing phase perturbation during gait. We hypothesized that, following a trip, altered timing, sequence, and magnitudes of perturbation-induced activations would emerge in the paretic and nonparetic support legs of stroke survivors compared to healthy control subjects. The swing foot was interrupted, while subjects walked on a treadmill. In healthy subjects, a sequence of perturbationinduced activations emerged in the contralateral stance leg with mean onset latencies of $87-147 \mathrm{~ms}$. The earliest latencies occurred in the hamstrings and hip abductor and adductors. The hamstrings, the adductor magnus, and the gastrocnemius dominated the relative balance of perturbation-induced activations. The sequence and balance of
\end{abstract}

Bahar Sharafi

bahar.sharafi@libertymutual.com

1 Liberty Mutual Research Institute for Safety, 71 Frankland Road, Hopkinton, MA 01748, USA

2 Department of Physical Medicine and Rehabilitation, Northwestern University, Chicago, IL, USA

3 Sensory Motor Performance Program, Rehabilitation Institute of Chicago, Chicago, IL, USA

4 Northwestern University Interdepartmental Neuroscience, Northwestern University, Chicago, IL, USA

5 Department of Biomedical Engineering, Northwestern University, Evanston, IL, USA activations were largely preserved after stroke. However, onset latencies were significantly delayed across most muscles in both paretic and nonparetic stance legs. The shortest latencies observed suggest the involvement of interlimb reflexes with supraspinal pathways. The preservation of the sequence and balance of activations may point to a centrally programmed postural response that is preserved after stroke, while post-stroke delays may suggest longer transmission times for interlimb reflexes.

Keywords Hemiparetic gait · Long-latency reflex · Interlimb reflex $\cdot$ Bilateral impairment $\cdot$ Reactive balance control

\section{Introduction}

Stroke survivors have significant residual deficits, related to balance control, as evidenced by a high rate of falls (Weerdesteyn et al. 2008). Walking in the real world often requires corrective reactions to recover or maintain balance in response to unexpected perturbations. Given that falls in stroke survivors most often occur while walking (Hyndman et al. 2002), reactive balance control during gait is critical to stroke survivors' ability to walk independently. Previous reports of post-stroke neuromuscular responses to postural perturbation have focused on balance recovery from static postures (e.g., Marigold and Eng 2006; Kirker et al. 2000). While decreased stability in response to gait perturbations has been documented after stroke (Krasovsky et al. 2013), investigation of the neuromuscular responses during the functional task of balance recovery while walking is lacking in this population. The goal of this work was to examine post-stroke deficits in the functional, neuromuscular responses that contribute to reactive balance control during gait. 
Emerging evidence suggests that chronic motor control impairments in post-stroke individuals involve abnormal multi-segmental behaviors. For example, abnormal coupling of stretch reflexes has been quantified under isometric conditions between hip adductor and knee extensor muscles in paretic legs of stroke survivors (Finley et al. 2008). We propose that, given the complex, bi-planar nature of balance control during gait, under destabilizing conditions, aberrant multi-joint neural constraints may impede the ability to generate appropriate neuromuscular responses.

To examine neuromuscular responses contributing to balance recovery during gait, we employed an induced trip. A trip involves interrupting the swing leg, necessitating a stabilizing neuromuscular response in the support leg (the stance leg contralateral to the perturbation). Immediately following a trip, prior to foot contact of the perturbed leg, the role of the support leg is to stabilize the body by arresting the angular momentum, resulting from the trip, and to provide time and clearance for the placement of the perturbed foot (Pijnappels et al. 2004). The focus of this study was to characterize the neuromuscular responses to a trip in the support leg. The initial single support phase of stabilization provides the opportunity to examine the stabilizing neuromuscular response in the paretic and nonparetic support legs in the absence of compensation from the opposite leg.

Previous studies have reported post-stroke delays of muscle activations in the paretic leg following postural perturbations from standing position (Marigold and Eng 2006; Kirker et al. 2000; Dietz and Berger 1984). During gait, bilateral delays were observed in an obstacle avoidance experimental paradigm (van Swigchem et al. 2013). Additionally, bilateral attenuation of interlimb cutaneous reflexes has been reported during gait (Zehr and Loadman 2012). While the origin of these post-stroke changes is unclear, examination under isolated single-joint constraints pointed to stroke-mediated impairments in interlimb spinal pathways (Stubbs et al. 2012). In light of these findings, and given the proposed role of interlimb reflexes in stumble correction during gait (e.g., Haridas et al. 2005), we hypothesized that altered timing, sequence, and magnitudes of perturbation-induced muscle activations would emerge in paretic and nonparetic support legs of stroke survivors compared to healthy control subjects.

\section{Methods}

\section{Subjects}

Eleven stroke survivors and eight healthy control subjects (Table 1) completed this study. Stroke survivors were recruited if they had a unilateral stroke at least 6 months prior to the test, hemiparesis of the lower limb, no cognitive deficits that would prevent them from performing the experimental protocol, and no prior history of injury or surgery to the lower limbs. All participants had to be able to walk for at least 10 min without wearing a brace, assistive device, body weight support, or reliance on treadmill handrails. Experimental procedures were approved by the Institutional Review Board of Northwestern University and complied with the principles of the Declaration of Helsinki. Informed consent was obtained prior to testing from all participants.

\section{Experimental setup and protocol}

Participants walked on an instrumented split-belt treadmill (Tecmachine, Andrézieux Bouthéon, France) at their selfselected fast speed. Subjects were instructed to walk at the fastest speed that they could maintain for continuous intervals, each lasting at least $10 \mathrm{~min}$, and in total adding up to at least $1 \mathrm{~h}$. This allowed the satisfaction of two criteria: (1) walking conditions were natural to each subject and (2) walking speed was fast enough that the interruption of the swing foot was challenging to the maintenance of balance. A custom code calculated the average swing phase duration for each subject based on the vertical ground reaction forces output by the treadmill force plates. A custom device (Shirota et al. 2014) induced trips by interrupting the foot via a retractable cable attached to the foot (Fig. 1). There was minimum slack in the cables while subjects walked so as not to get caught under the feet. To interrupt the foot, a solenoid valve closed a flap over the cable attached to the foot for $150 \mathrm{~ms}$. Each cable had a uniaxial load cell to measure the trip force (force required to interrupt the foot). Closing of the solenoid valves was timed so that trip onset, defined as the moment at which trip force exceeded $10 \mathrm{~N}$, occurred between 10 and $30 \%$ of swing. Early swing phase was chosen for the perturbation to allow for a longer single support phase during the initial phase of stabilization.

To minimize anticipation of the trip, perturbations were applied to both feet in random order and at random intervals, at least $1 \mathrm{~min}$ apart, and participants were engaged in conversation while they walked. The length of each recorded trial was $10 \mathrm{~s}$ and included at least two unperturbed strides prior to the trip. Participants wore a safety harness to prevent falling in case of unsuccessful recovery. The harness cable had sufficient slack so as not to interfere unless a fall was imminent. Participants were allowed to grab the treadmill handrails in case of a fall; however, they were advised to avoid touching the handrails unless absolutely necessary following a trip. 
Table 1 Subject information

\begin{tabular}{|c|c|c|c|c|c|c|c|c|c|c|c|c|}
\hline Subject & $\begin{array}{l}\text { Age } \\
\text { (years) }\end{array}$ & Sex & $\begin{array}{l}\text { Height } \\
(\mathrm{cm})\end{array}$ & $\begin{array}{l}\text { Weight } \\
(\mathrm{kg})\end{array}$ & $\begin{array}{l}\text { Lesion } \\
\text { side }\end{array}$ & $\begin{array}{l}\text { Months } \\
\text { post- } \\
\text { stroke }\end{array}$ & $\begin{array}{l}\text { Stroke } \\
\text { type }^{\mathrm{a}}\end{array}$ & $\begin{array}{l}\text { Berg } \\
(56)\end{array}$ & $\begin{array}{l}\text { LMFM }^{\mathrm{a}} \\
(34)\end{array}$ & $\begin{array}{l}\text { SPFM }^{\mathrm{a}} \\
(24)\end{array}$ & $\begin{array}{l}\text { MMSE }^{a} \\
(30)\end{array}$ & $\begin{array}{l}\text { Speed } \\
(\mathrm{m} / \mathrm{s})\end{array}$ \\
\hline S1 & 52 & $\mathrm{~F}$ & 162 & 63 & $\mathrm{~L}$ & 35 & $\mathrm{H}$ & 51 & 18 & & 30 & 0.3 \\
\hline $\mathrm{S} 2$ & 53 & $\mathrm{~F}$ & 168 & 66 & $\mathrm{~L}$ & 36 & $\mathrm{H}$ & 49 & 24 & & & 0.6 \\
\hline S3 & 58 & M & 180 & 78 & $\mathrm{R}$ & 55 & I & 49 & 22 & 24 & 21 & 0.6 \\
\hline $\mathrm{S} 4$ & 58 & M & 188 & 90 & $\mathrm{~L}$ & 75 & I & 51 & 22 & 24 & & 0.6 \\
\hline S5 & 65 & $\mathrm{~F}$ & 157 & 57 & $\mathrm{~L}$ & 117 & I & 41 & 17 & 24 & & 0.5 \\
\hline S6 & 58 & M & 185 & 92 & $\mathrm{~L}$ & 102 & I & 45 & 22 & & 30 & 0.5 \\
\hline S7 & 57 & M & 180 & 107 & $\mathrm{R}$ & 76 & $\mathrm{H}$ & 54 & 26 & 24 & 30 & 0.9 \\
\hline S8 & 57 & M & 173 & 83 & $\mathrm{~L}$ & 33 & & 50 & 24 & 24 & 29 & 0.9 \\
\hline S9 & 48 & M & 165 & 93 & $\mathrm{R}$ & 184 & & 46 & 22 & 18 & 29 & 0.4 \\
\hline $\mathrm{S} 10$ & 42 & M & 175 & 84 & $\mathrm{~L}$ & 82 & & 56 & 26 & 24 & 28 & 1.1 \\
\hline $\mathrm{S} 11$ & 55 & M & 173 & 84 & L & 15 & I & 49 & 16 & & 29 & 0.6 \\
\hline $\mathrm{C} 1$ & 51 & M & 183 & & & & & & & & & 1.3 \\
\hline $\mathrm{C} 2$ & 52 & M & 183 & & & & & & & & & 1.1 \\
\hline $\mathrm{C} 3$ & 51 & M & 175 & & & & & & & & & 1.3 \\
\hline $\mathrm{C} 4$ & 44 & M & 178 & & & & & & & & & 1.4 \\
\hline C5 & 46 & $\mathrm{~F}$ & 152 & & & & & & & & & 1.2 \\
\hline C6 & 51 & $\mathrm{~F}$ & 173 & & & & & & & & & 1.4 \\
\hline C7 & 59 & F & 168 & & & & & & & & & 1.1 \\
\hline C8 & 41 & $\mathrm{~F}$ & 152 & & & & & & & & & 1.3 \\
\hline
\end{tabular}

Perfect scores are shown in parentheses

$S$ post-stroke subject identifiers, $C$ control subject identifiers, $H$ hemorrhagic, $I$ ischemic, $L M F M$ Fugl-Meyer (lower limb motor), $S P F M$ FuglMeyer (sensation and proprioception), MMSE mini-mental state evaluation

${ }^{a}$ Missing entries indicate unavailable values. Speed is self-selected fast walking speed

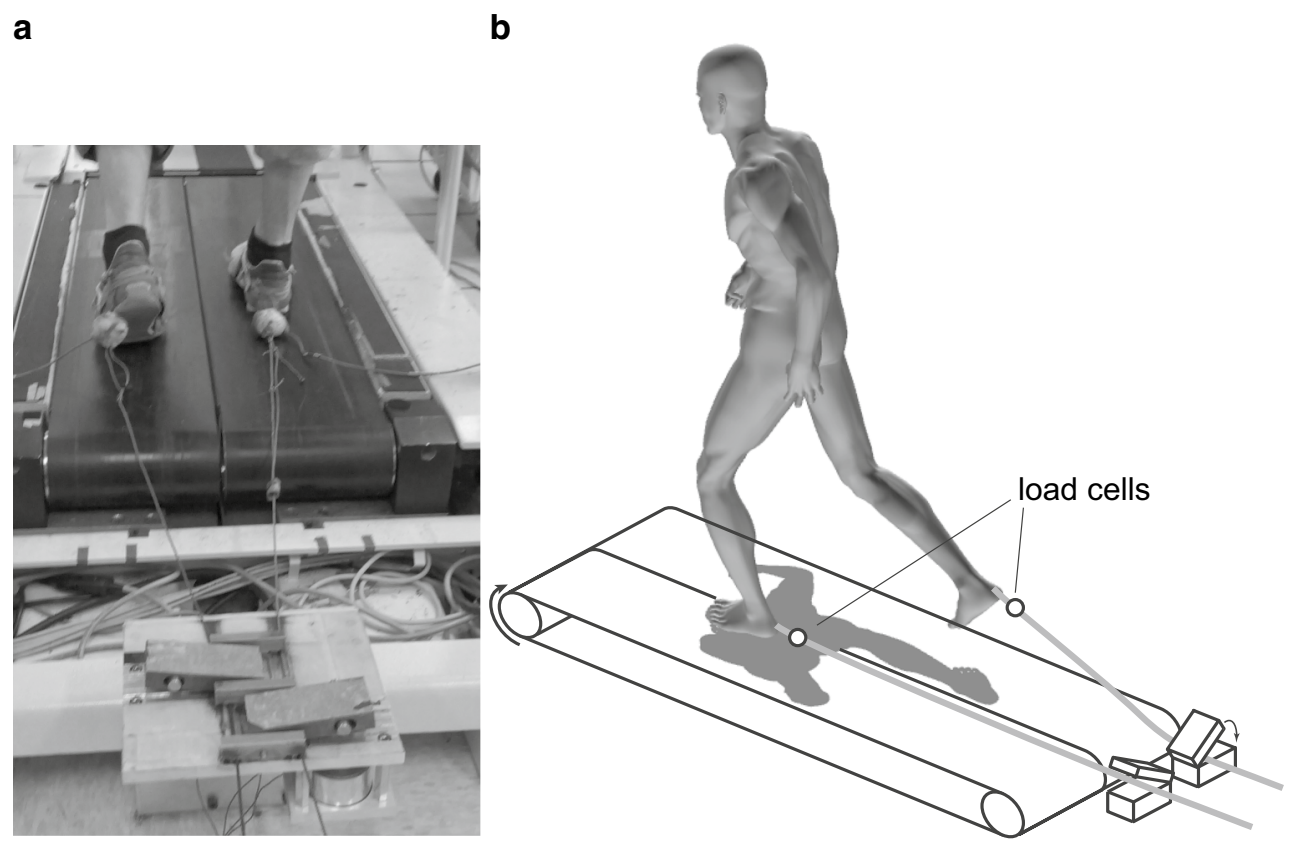

Fig. 1 Subjects walked on an instrumented split-belt treadmill. Trips where induced by catching and holding the retractable cable attached to either foot during swing phase using a custom-made device. Photograph (a) and schematic (b) of the setup are shown 


\section{Data collection}

Gait kinematics were recorded using an eight-camera motion capture system (Motion Analysis Corporation, Santa Rosa, CA, USA). Forty-nine reflective markers were placed on each participant. Marker trajectories were recorded at $100 \mathrm{~Hz}$. Surface EMG (Delsys, Boston, MA, USA) was recorded bilaterally from the soleus (SOL), medial gastrocnemius (MGAS), peroneus longus (PL), tibialis anterior (TA), semitendinosus (ST), biceps femoris $(\mathrm{BF})$, vastus medialis $(\mathrm{VM})$, vastus lateralis $(\mathrm{VL})$, rectus femoris (RF), adductor magnus (ADD), gluteus maximus (GMA), and gluteus medius (GME). Ground reaction forces, trip force, harness force, and EMG were sampled at $1000 \mathrm{~Hz}$. The raw EMG signal from each muscle was highpass filtered at $250 \mathrm{~Hz}$, rectified, then low-pass filtered at $100 \mathrm{~Hz}$ with a zero-phase fourth-order Butterworth filter. Trip force and ground reaction forces were filtered at 30 and $15 \mathrm{~Hz}$, respectively. All trials were recorded on video to visually determine the incidence of handrail use. A trial was categorized as a failed recovery attempt if the harness was engaged with a load greater than $10 \%$ of body weight or if the participant was unable to recover without grabbing the handrail.

\section{Kinematic onsets}

Onset latencies of kinematic events from trip onset were determined as follows. Joint angle trajectories, output by Cortex system (Motion Analysis Corporation, Santa Rosa, CA, USA), were low-pass filtered at $10 \mathrm{~Hz}$ using a fourthorder Butterworth filter. Mean profiles were calculated from all unperturbed strides and subtracted from individual perturbed strides. To establish a baseline level and a baseline level of variability, five data points $(50 \mathrm{~ms})$ of the subtracted profile, prior to perturbation onset, were used to calculate a mean and SD. An onset of deviation was detected if the subtracted value, averaged over four data points (40 ms), was more than 4 SD away from the baseline level.

\section{EMG onsets}

EMG onset latencies were calculated relative to trip force onset. Alternatively, latencies could have been reported relative to the earliest detected onset of joint angle deviation, i.e., ankle dorsiflexion. Given the similarity of ankle perturbation onsets across groups, this method would not have altered our findings (results). EMG time histories from all trials were conditioned using the Teager-Kaiser energy operator (Solnik et al. 2010). For each subject and each muscle, EMG mean and SD were calculated for all unperturbed strides aligned from heel strike. The mean unperturbed EMG was subtracted from individual perturbed strides aligned from the heel strike of the support leg. A perturbation-induced onset was determined if the subtracted, conditioned EMG, averaged over $20 \mathrm{~ms}$, exceeded 4 SD of the conditioned EMG at the corresponding point in the unperturbed gait cycle. This threshold was based on a sensitivity analysis of a subset of post-stroke and healthy EMG time series and was chosen to maximize agreement with expert visual detection and minimize the number of false positives. Onset latencies detected earlier than $30 \mathrm{~ms}$ from trip onset were designated as false positives, given that neuromuscular latencies in the contralateral leg cannot be shorter than a monosynaptic stretch reflex.

\section{Similarity of EMG onset sequence}

For each support leg condition (control, nonparetic, and paretic), we defined the mean sequence of perturbationinduced activations as a vector, $V_{12 \times 1}$, containing the respective mean onset latencies. To evaluate the similarity between the mean sequence in the paretic and nonparetic support legs with that in the control support leg, we calculated the Euclidean distance, $d$, between this vector, for each condition, with that of the control condition, $V_{\mathrm{c}}$, as follows: $d_{\mathrm{np}}=\sqrt{\left(V_{\mathrm{np}}-V_{\mathrm{c}}\right)^{\mathrm{T}} \cdot\left(V_{\mathrm{np}}-V_{\mathrm{c}}\right)}$ and $d_{n}=\sqrt{\left(V_{\mathrm{p}}-V_{\mathrm{c}}\right)^{\mathrm{T}} \cdot\left(V_{\mathrm{p}}-V_{\mathrm{c}}\right)}$, where the subscript np denotes nonparetic and $\mathrm{p}$ denotes paretic. If $d$ was significantly smaller than the distance expected if the sequence was generated at random (e.g., d'Avella and Bizzi 2005), we concluded that the mean sequence of perturbationinduced activations for that support leg condition (paretic or nonparetic) was similar to the mean control sequence. To perform this comparison, we generated a set of $10^{4}$ vectors, $V_{\text {random }}^{(i)}$, by randomly permutating the elements of $V$. We calculated the Euclidean distance, $d_{\text {random }}^{(i)}$, between each $V_{\text {random }}^{(i)}$ and the mean control sequence, $V_{\mathrm{c}}$. From a 100 bin histogram of $d_{\text {random }}^{(i)}$ values, we generated an empirical probability density function of distances of randomly generated onset sequences for each limb condition. We determined the $p$ value for rejecting the null hypothesis that the actual distance, $d$, was drawn from this distribution by integrating the empirical probability density function from 0 to $d$.

\section{EMG magnitudes}

To determine the magnitude of perturbation-induced activations across muscles as a function of time after perturbation, we subtracted the mean of band-passed, rectified, and low-passed EMG of unperturbed stance phase from the mean EMG of the support leg during perturbed trials. To enable comparison across subjects, the subtracted EMG was 
Table 2 Trip onset, force, perturbed ankle stiffness, and swing leg kinematic onsets for perturbations applied to control, nonparetic, and paretic swing legs

\begin{tabular}{|c|c|c|c|c|c|c|}
\hline & \multicolumn{3}{|l|}{ Swing leg } & \multicolumn{3}{|l|}{$p$ values } \\
\hline & Control & Nonparetic & Paretic & $\begin{array}{l}\text { Control versus } \\
\text { nonparetic }\end{array}$ & $\begin{array}{l}\text { Control versus } \\
\text { paretic }\end{array}$ & $\begin{array}{l}\text { Nonparetic } \\
\text { versus paretic }\end{array}$ \\
\hline Peak trip force & $203.8 \pm 9.8$ & $146.5 \pm 7.5$ & $107.8 \pm 18.8$ & $<0.001$ & $<0.001$ & 0.014 \\
\hline $\begin{array}{l}\text { Trip onset (\% of average } \\
\text { swing duration) }\end{array}$ & $25.8 \pm 0.6$ & $24.0 \pm 1.0$ & $20.8 \pm 1.4$ & & 0.006 & 0.03 \\
\hline Ankle stiffness $\left(\mathrm{Nm} /{ }^{\circ}\right)$ & $0.66 \pm 0.03$ & $0.76 \pm 0.12$ & $1.37 \pm 0.57$ & & & \\
\hline $\begin{array}{l}\text { Onset ankle dorsiflexion } \\
\text { (ms) }\end{array}$ & $27.1 \pm 0.4$ & $25.3 \pm 0.5$ & $24.7 \pm 0.7$ & & & \\
\hline Onset knee flexion (ms) & $86.5 \pm 3.0$ & $77.3 \pm 1.8$ & $83.9 \pm 3.2$ & & & \\
\hline Onset hip extension (ms) & $64.2 \pm 1.8$ & $74.3 \pm 1.0$ & $102.5 \pm 3.0$ & & $<0.001$ & 0.03 \\
\hline
\end{tabular}

Trip onset was defined as the moment at which trip force exceeded $10 \mathrm{~N}$. Values are intragroup mean \pm SE of the mean. Only $p$ values that show significant differences between support leg conditions $(p<0.05)$ have been presented

normalized by the peak of mean EMG of unperturbed gait cycles. Normalized, subtracted EMG profiles were averaged within groups. Within-group average time series were then time-averaged by binning a 180 -ms post-perturbation interval into $20 \mathrm{~ms}$ bins. Onset and magnitude data for a given support leg condition were only presented for muscles that activated in more than of $50 \%$ of trips across all subjects.

\section{Between-group comparisons}

Comparisons across support leg conditions (control, nonparetic, and paretic) were made using repeated-measures mixed-effect models (Littell et al. 1998). The value from each trial was considered a repeated measure associated with the corresponding subject. A compound symmetry correlation structure was used to take into account correlations within each subject. All statistical analyses were performed in SAS 9.2 (SAS Institute Inc., Cary, NC, USA).

\section{Analyses of confounding effects}

The following variables were examined as covariates ("Appendix"): walking speed, perturbed ankle stiffness, and support leg posture at trip onset.

\section{Results}

Post-stroke and control subjects walked at average speeds of $0.64 \pm 0.07 \mathrm{~m} / \mathrm{s}$ (mean $\pm \mathrm{SE}$ ) and $1.26 \pm 0.04 \mathrm{~m} / \mathrm{s}$, respectively. Between eight and twenty-eight trips (16 mean \pm 5
SD) were successfully recorded for each leg. Perturbations of the paretic swing leg were successfully recorded in 10 of the 11 post-stroke subjects. A total of 128,164 , and 159 perturbations of control, nonparetic, and paretic swing legs were successfully recorded. Mean trip onsets and trip forces are presented in Table 2. Of the total number of perturbed trials for the control, nonparetic, and paretic swing legs (corresponding to control, paretic, and nonparetic support legs), 26, 68, and $33 \%$, respectively, resulted in failed recovery attempts.

\section{Onsets of kinematic events}

The interruption of the swing foot induced a deviation of swing leg joint angles from their unperturbed trajectory. The perturbation increased the dorsiflexion of the ankle and impeded the extension and flexion of the knee and hip, respectively. Perturbation-induced onsets of joint angle deviations in control, nonparetic, and paretic swing legs are given in Table 2. No significant differences were found between limb conditions in the onset of deviations of the ankle and knee flexion angles. The onset of hip extension was significantly later in the paretic perturbed hip compared to nonparetic and control conditions. Representative swing leg kinematics and support leg EMG are shown in Figs. 2 and 3, respectively.

\section{EMG onsets}

In control subjects, the earliest EMG onsets in the support leg occurred in the hamstrings (BF and ST), the hip adductor (ADD), and abductor (GME), with intragroup mean 

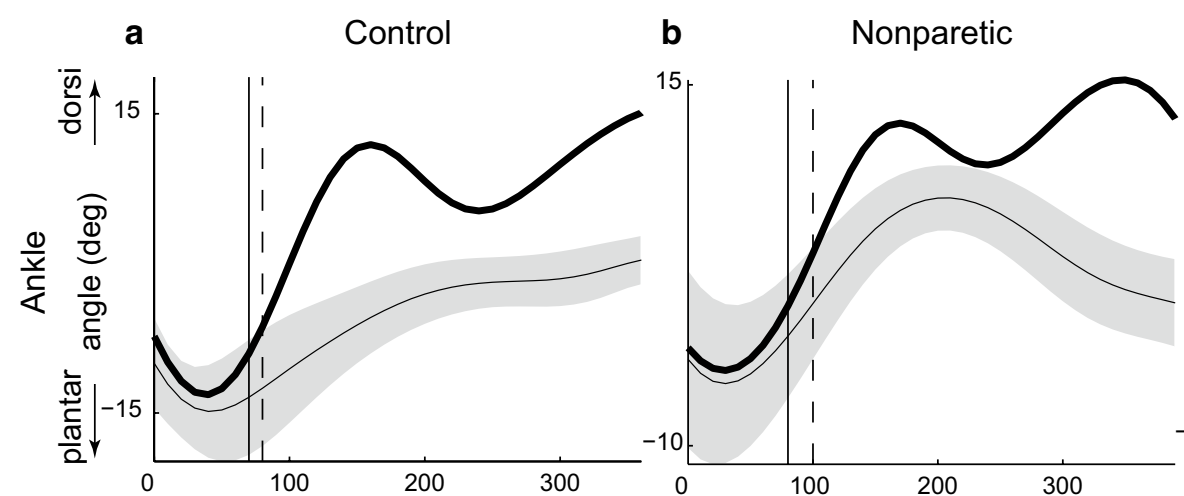

C Paretic
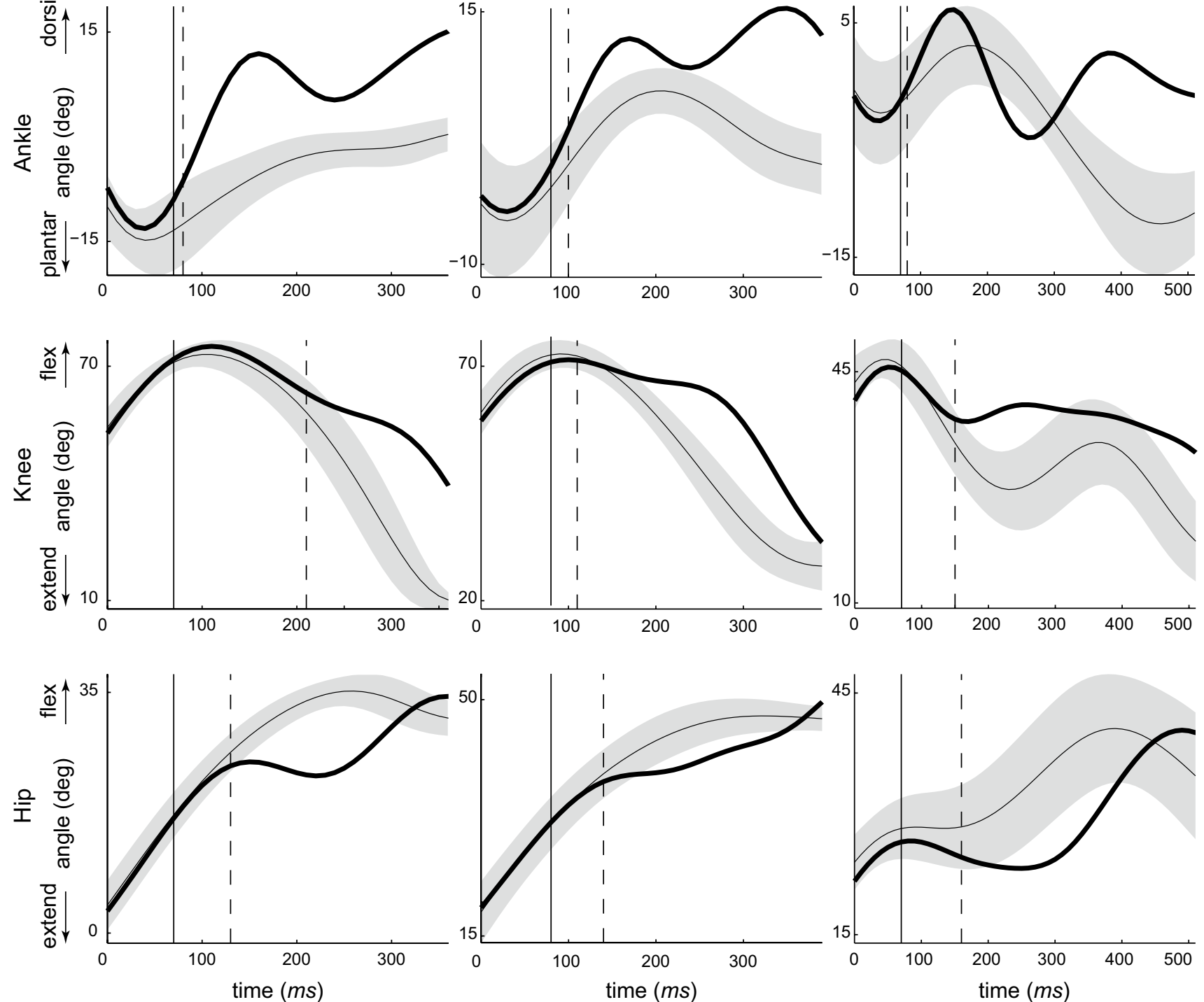

Fig. 2 Representative swing phase kinematics are shown from a control subject (a) and a stroke survivor (b, c). The thin solid curve and shaded area represent the mean and 2 SD of unperturbed swing phase trajectory. The thick solid curve depicts the joint trajectories of the

perturbed swing leg in one trip trial. Solid vertical line denotes trip onset. Dashed vertical line denotes the detected onset of joint angle deviation

latencies of 87-96 ms from trip onset. These were followed by responses in the quadriceps (VM, VL, and RF) with mean latencies of 101-113 ms, the GMA (mean latency of $117 \mathrm{~ms}$ ), and the MGAS (mean latency of $119 \mathrm{~ms}$ ). The TA had a mean latency of $147 \mathrm{~ms}$. The SOL and the PL were activated in less than $50 \%$ of trips across control subjects. In paretic and nonparetic support legs, the earliest onsets appeared in the BF, ST, ADD, and GME muscles. However, the mean onset latencies of these muscles were delayed by $20-50 \mathrm{~ms}$ in the paretic leg. All proximal muscles (hamstrings, quadriceps, gluteals, and adductor magnus) had significantly delayed onsets in both paretic and nonparetic legs compared to control subjects. Significant differences between paretic and nonparetic legs were only found in the quadriceps, with longer onset latencies in the nonparetic quadriceps compared to the paretic (Fig. 4).

\section{Similarity of EMG onset sequence}

The paretic support leg expressed a similar sequence of activations to the control support leg. We rejected the null hypothesis that $d_{\mathrm{p}}$, the Euclidean distance between the mean paretic sequence and the mean control sequence, was drawn from the distribution of randomly generated sequences 

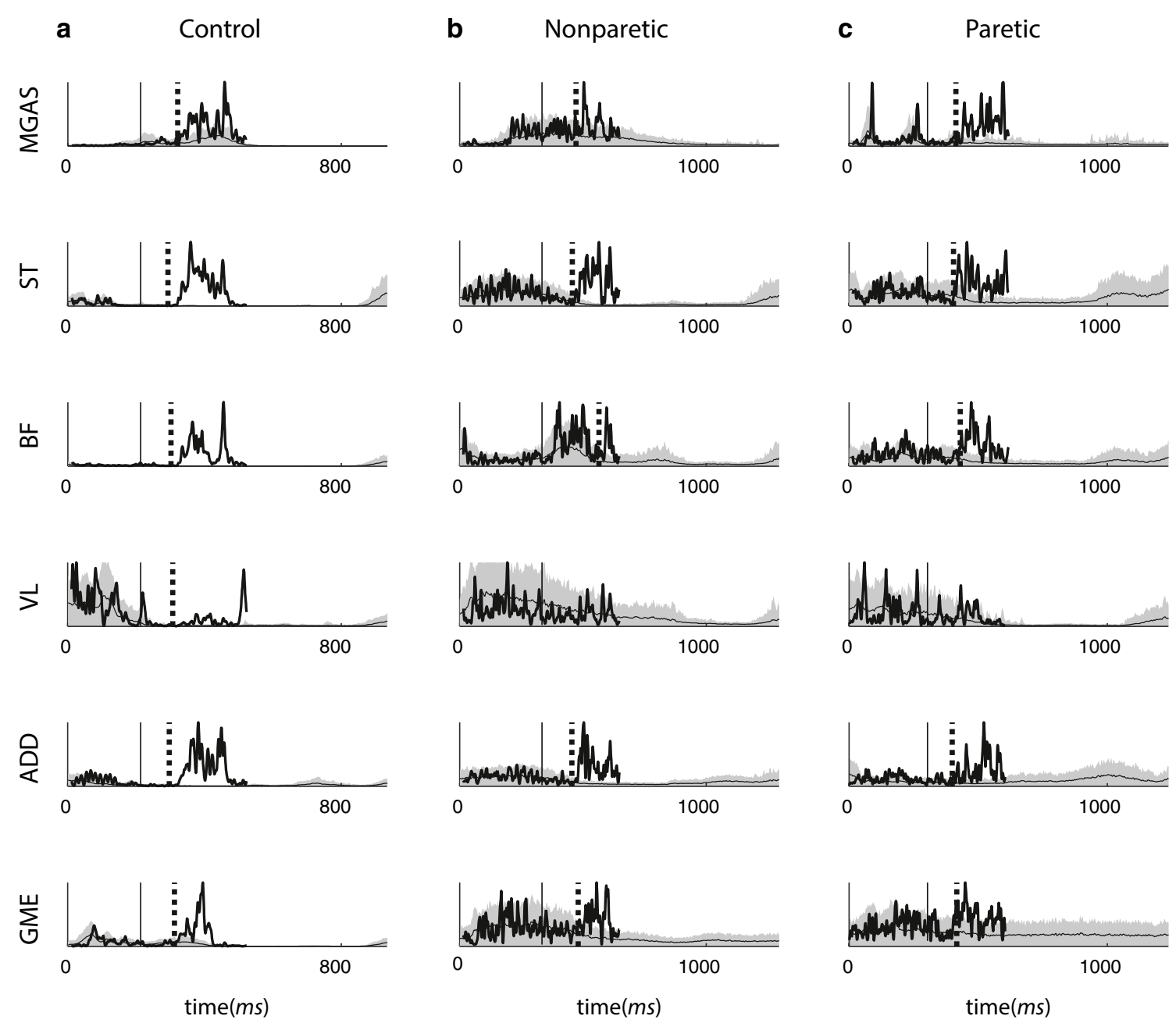

Fig. 3 Representative support leg rectified and filtered EMG time series are shown from a number of muscles for a control subject (a) and a stroke survivor $(\mathbf{b}, \mathbf{c})$. The thin solid curve and shaded area represent the mean and $2 \mathrm{SD}$ of unperturbed EMG. The thick solid curve depicts the EMG of the support leg in one trip trial. Solid vertical line

$(p=0.014)$. In other words, the mean paretic sequence was significantly closer to the mean control sequence than can be expected if the paretic onset latencies were randomly ordered between muscles. In the nonparetic leg, although the distance with the control sequence, $d_{\mathrm{np}}$, was smaller than the mean distance expected at random, the null hypothesis could not be rejected $(p=0.28)$. This discrepancy arose from the long delays in the onsets of the nonparetic quadriceps. In fact, once quadriceps were eliminated from the analysis, the sequence of activations in the nonparetic support leg was significantly similar to the control support leg $(p=0.006)$.

\section{EMG magnitudes}

Following the trip, the relative balance of magnitudes of perturbation-induced muscle activations in the support leg was denotes trip onset. Dashed vertical line denotes the detected onset of perturbation-induced EMG activity in the muscle. Time zero is the heel strike of the support leg. The vertical axis is in $\mathrm{mV}$ and is not on the same scale for all plots

dominated by the ST, BF, ADD, and MGAS, across all groups (Fig. 5). Magnitudes of normalized perturbation-induced activations were larger in the control ST, BF, ADD, and GME compared to the paretic and nonparetic legs (Fig. 5). Magnitudes of normalized perturbation-induced activations were larger in the nonparetic ST, BF, and ADD compared to the paretic leg, although significance was not reached.

\section{Discussion}

The goal of this study was to determine whether timing, sequence, and balance of perturbation-induced muscle activations, in response to a unilateral gait perturbation, were altered in the paretic and nonparetic support legs of stroke survivors relative to healthy subjects. We found a largely 


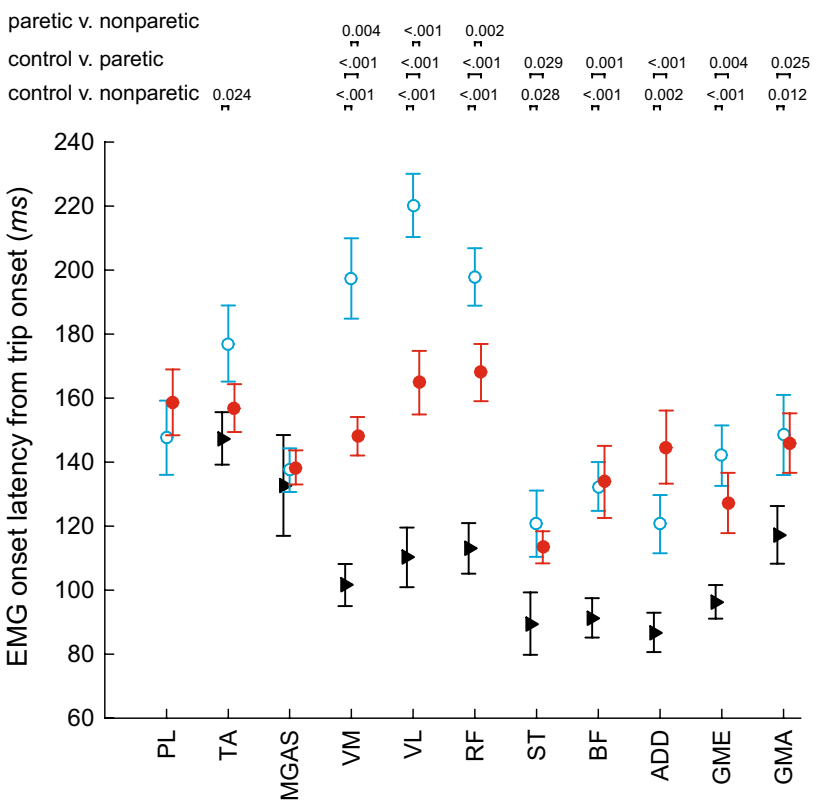

Fig. 4 Mean EMG onset latencies were calculated for each group from trip onset. Error bars represent SE. $p$ values smaller than 0.05 are shown for comparison of EMG onset latencies between control and nonparetic, control and paretic, and paretic and nonparetic support legs. In each group, only muscles that activated in more than an average of $50 \%$ of trip trials across members of that group are shown. For example, the soleus was activated in an average of 43,47 , and $48 \%$ of trials in the control, nonparetic, and paretic support legs, respectively

similar sequence and balance of activations across paretic, nonparetic, and control support legs. The earliest perturbation-induced responses in the support leg appeared in the hamstrings and hip adductor and abductors. These muscles along with the gastrocnemius dominated the relative balance of activations. However, onsets of neuromuscular responses in the support leg were significantly delayed in stroke survivors compared to healthy control subjects, irrespective of whether the paretic or the nonparetic leg was perturbed.

Onset latencies reported here for control subjects were generally comparable to previous reports in ST, BF, RF, and VL, in a limited number of studies (Eng et al. 1994; Schillings et al. 2000; Pijnappels et al. 2005a). However, the onset latency of MGAS was significantly longer compared to these reports (Eng et al. 1994; Pijnappels et al. 2005a). Key differences in the experimental paradigm may have contributed to this discrepancy: (1) In the present study, the foot was interrupted using a flexible cable that decelerated the foot within an approximately 40-ms interval from trip onset, whereas the impact of the foot with a relatively rigid obstacle, as in the above studies, would have instantaneously stopped the forward movement of the foot. (2) In the above studies, the impact of the foot with an obstacle, in early swing phase, would have plantarflexed the ankle. In this study, the imposed

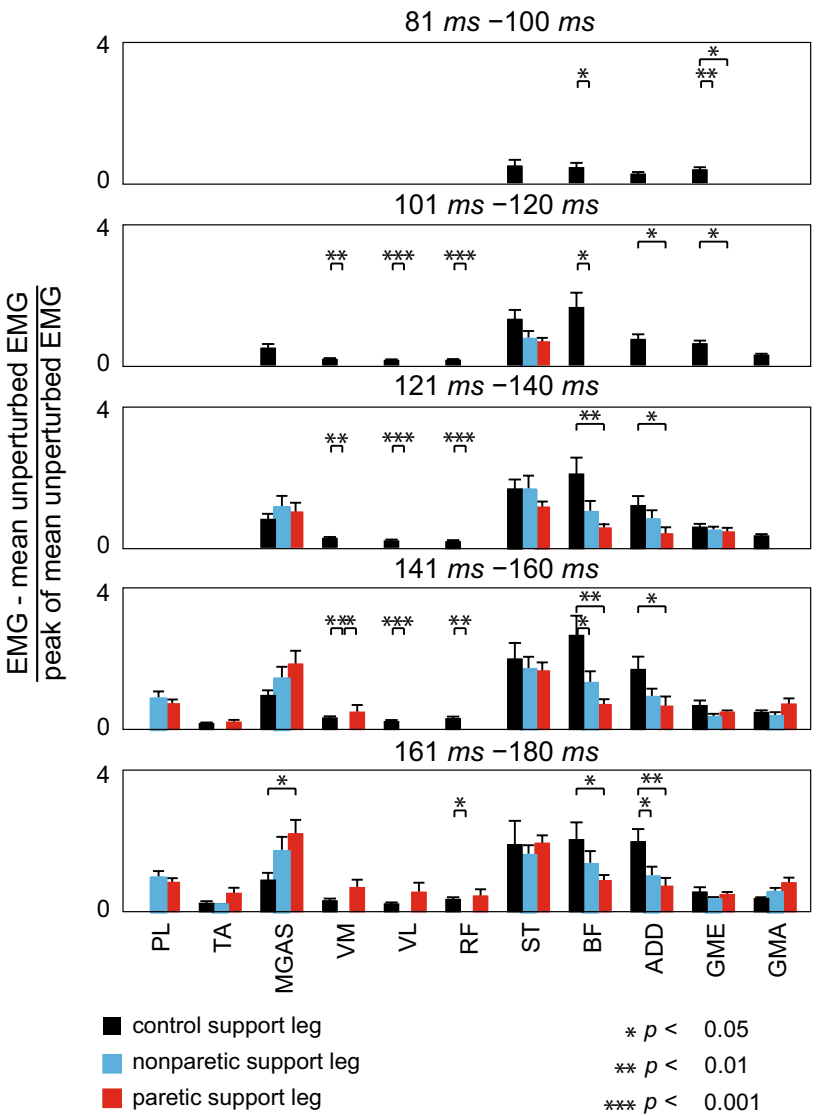

Fig. 5 Mean EMG of unperturbed stance was subtracted from mean EMG of trip strides for the support leg and normalized by the peak mean EMG of unperturbed gait cycles for each subject. Group mean subtracted and normalized EMG was averaged for 20-ms intervals following trip onset for a $180-\mathrm{ms}$ period. Nonzero mean magnitudes are shown only in intervals greater than the group mean onset latency of each muscle (Fig. 4). Error bars represent SE

perturbation dorsiflexed the swing ankle. It is possible that intralimb and interlimb reflexes, triggered by the perturbation imposed at the ankle, are direction-dependent. For instance, although we found no such comparison in healthy individuals, in spinal cord injured patients, the nature of ipsilateral heteronymous reflexes was strongly dependent on whether a dorsiflexion or a plantarflexion rotation was imposed at the ankle (Schmit et al. 2000). Given that early responses in the support leg can be largely attributed to the perturbation experienced by the swing leg (see "Nature of the observed responses", below), it is likely that these differences in the applied perturbation resulted in differences in the timing of support leg neuromuscular responses.

\section{Nature of the observed responses}

While we did not aim to directly address the underlying pathways mediating the responses, we made the following 
observations. The onset latencies of responses in the control group, with the possible exception of the TA, were below reported thresholds for voluntary reactions (Swinnen et al. 1995), suggesting the automatic nature of responses. It has been proposed that latencies of these orders of magnitude reflect the involvement of polysynaptic supraspinal pathways (Nielsen et al. 1997). A recent examination of interlimb reflexes following unilateral perturbation confirmed the contribution of a transcortical loop to observed responses (mean latency of $76 \mathrm{~ms}$ ) (Stevenson et al. 2013).

The exact mechanisms contributing to the contralateral leg response may be multifactorial, including muscle afferents at the joints of the perturbed swing leg, local stretch reflexes at the joints of the support leg, vestibular postural reflexes, and cutaneous afferents from the support foot. The earliest detectable deviation in support leg joint angles appeared later than the onset of muscle activity in that leg. Therefore, it is unlikely that the observed response in the support leg was due to muscle afferents in the same leg. Moreover, EMG onsets preceded any detected deviation in the acceleration of the head or changes in the trajectory of the center of pressure, precluding the involvement of vestibular postural reflexes and cutaneous afferents from the support foot, in the early part of the response. Taken together, these observations suggest that the reflex responses in the support leg may have been triggered by muscle afferents from the perturbed leg. Muscle afferents at any of the joints of the perturbed leg may have contributed to neuromuscular responses in the support leg. Given the latencies of crossed reflexes, especially with the possible involvement of transcortical pathways (Stevenson et al. 2013), it is likely that the reported onsets, in a majority of muscles, were triggered due to muscle afferents at the swing ankle rather than the hip and knee joints.

\section{Preserved sequence and balance of perturbation-induced EMG after stroke}

The sequence and balance of activation in the paretic and nonparetic support legs were largely consistent with those observed in control subjects. One might argue that the activation of the hamstrings and the hip adductor and abductors may comprise a centrally programmed activation synergy, forming a "first line of defense" on the support side. Our results suggest that this first line of defense is largely preserved following stroke. It has been suggested that postural responses are centrally programmed as a set of synergies, or activation patterns, with the selection of the appropriate pattern triggered by afferent input (Dietz 1992). This argument is supported by feline models that reveal the existence of a centrally coordinated postural response involving the reticular formation (Stapley and Drew 2009). It is conceivable that similar pathways contribute to the responses observed in humans.

\section{Bilateral delays in reflex onsets after stroke}

Onsets of neuromuscular responses in the support leg were significantly delayed in a majority of muscles in stroke patients relative to control subjects. These onsets were similar between paretic and nonparetic support legs, with significant differences detected only in the quadriceps. Bilateral post-stroke impairment in the modulation of interlimb reflexes has previously been reported in response to cutaneous nerve stimulation (Stubbs et al. 2012; Zehr and Loadman 2012). Additionally, bilateral delays were reported in onsets of muscle activations when performing within-step adjustments (van Swigchem et al. 2013). This study characterized the effect of stroke on the timing and magnitudes of neuromuscular responses in the context of the functional task of balance recovery during gait. In response to perturbations from standing, delayed responses were only reported in the paretic leg contralateral to a perturbation (Dietz and Berger 1984). The presence of bilateral delays during gait may be due to the different nature of balance control during gait compared to standing. Following a perturbation from standing position, muscle activations follow a distal-to-proximal sequence and are limited to muscles acting in the plane of the perturbation. During gait, the reactive control of balance requires the cross-planar activation of muscles and involves a proximal-to-distal sequence. It is possible that different neural circuitries are engaged in the control of posture during gait compared to standing.

\section{Delayed knee stabilization in the nonparetic leg}

Onset latencies of the quadriceps were significantly longer in the nonparetic compared to the paretic support leg. It could be argued that this asymmetry resulted from differences between afferent inputs from the perturbed paretic and nonparetic legs. If this were the case, we would expect this asymmetry to emerge across all muscles. Alternatively, one might argue that the asymmetrical latencies of the quadriceps are related to differences in the kinematics of the perturbed paretic and nonparetic swing legs. Onsets of perturbation-induced deviation were similar for nonparetic and paretic swing knees. However, while the nonparetic knee continued to extend, the paretic knee went into flexion (Fig. 2). Given the longer latencies of the quadriceps in the stroke group, differences in perturbation-induced knee kinematics between the paretic and nonparetic sides may have contributed to the difference in latencies of these muscles.

\section{Functional role of the observed responses}

Our results extend the sagittal plane findings reported in healthy subjects (Pijnappels et al. 2005b; Eng et al. 1994; Schillings et al. 2000). We found that the hip adductors 
and abductors, along with the hamstrings, had the shortest response latencies and dominated the relative balance of activations during the early recovery period. This finding suggests that the motor control system prioritizes frontal plane stabilization, even in response to sagittal plane perturbations.

\section{Functional and clinical implications}

Longer reflex latencies may be a limiting factor for stroke survivors' ability to avoid falls in response to gait perturbations. While recovery from a trip can occur over multiple steps and is dependent on responses in both legs, delayed early responses in the support leg may result in excessive angular momentum and the inability to appropriately place the perturbed foot, thereby limiting the ability to arrest the forward directed angular momentum in subsequent steps.

Conversely, the cross-planar sequence and balance of perturbation-induced activations in stroke survivors were similar to healthy control subjects. The timing of poststroke standing postural reflexes was shown to be modifiable (Marigold et al. 2005). Further studies are needed to determine whether improvements in response latencies may also be achieved under dynamic conditions. Finally, bilateral delays in the neuromuscular response measured in the present study support the argument that there is no unaffected side after stroke and highlight the importance of focusing rehabilitation efforts on both limbs.

\section{Limitations and confounding effects}

We sought to examine functional responses to a destabilizing perturbation under natural walking conditions; therefore, subjects were instructed to walk at their self-selected fast speed. This meant that stroke survivors walked at speeds significantly slower than control subjects, potentially confounding the across-group comparisons. However, we found no significant effect of walking speed on onset latencies ("Appendix"). This finding was consistent with earlier literature. While changes in reflex size were observed with walking speed (Sinkjaer et al. 1996), no speed dependence was reported for the latencies of short-, medium-, or long-latency reflexes (Sinkjaer et al. 1996, 1999; Cronin et al. 2009; Grey et al. 2001). Additionally, it could be argued that, in the current experiment, differences in walking speed resulted in differences in perturbation velocity, which may have altered onset latencies. However, previous studies did not find onset latencies to be dependent on perturbation velocity (e.g., Cronin et al. 2009; Finley et al. 2013).

Characteristic differences between the populations in limb posture, joint stiffness, and background activation could also potentially confound the results. It could be argued that delayed EMG onsets in stroke survivors compared to control subjects may be accounted for by differences in these variables. However, we found no significant effect of support leg posture and perturbed ankle stiffness on onset latencies ("Appendix"). Additionally, examination of the literature yielded no evidence that onset delays, of magnitudes observed here, could be attributed to differences in background activation. A number of studies have shown no change in onset latency as a function of background activation for short-, medium-, and long-latency stretch reflexes in single-joint constrained experiments (Toft et al. 1991, 1989; Finley et al. 2012; Kimura et al. 2003). While some decrease in peak or onset latency has been reported with several-fold increases in background activation (Toft et al. 1989; Ogiso et al. 2002), these changes were much smaller (1-9 ms) than differences between stroke and control groups (20-60 ms). Taken together, these reports combined with the result of our analysis of the effect of covariates suggest that post-stroke onset delays observed in this study did not arise from differences in the above confounding effects. Rather, onset delays likely had neurophysiological origins.

Finally, the goal of this study was to examine changes in post-stroke neuromuscular responses in the stabilizing support leg. To examine the underlying mechanisms of these changes, statistical analyses of the relationship of joint kinematics and EMG in the perturbed leg to the neuromuscular responses in the support leg will be informative. In addition, the duration of the perturbation-induced response and the presence of co-activation are some factors, other than onset delays, that could contribute to impaired balance recovery. These factors will be examined in future investigations.

Acknowledgments We are grateful to Camila Shirota for the use of her custom-made tripping device and assistance with the experimental setup, to Heidi Roth for help with subject recruitment and evaluation, and to Santosh Verma for assistance with statistical analyses. Funding for this work was provided by National Institute of Neurological Disorders and Stroke, Grant 5R01NS064084-02, and National Institute on Disability and Rehabilitation Research, Grant H133P110013-12.

Open Access This article is distributed under the terms of the Creative Commons Attribution 4.0 International License (http://creativecommons.org/licenses/by/4.0/), which permits unrestricted use, distribution, and reproduction in any medium, provided you give appropriate credit to the original author(s) and the source, provide a link to the Creative Commons license, and indicate if changes were made.

\section{Appendix: Analyses of confounding effects}

We examined the effect of walking speed, support leg posture, and perturbed ankle stiffness, by including these variables as covariates in repeated-measures mixed-effect 
Table 3 Stance leg posture

\begin{tabular}{|c|c|c|c|c|c|c|}
\hline & \multicolumn{3}{|l|}{ Stance leg } & \multicolumn{3}{|l|}{$p$ values } \\
\hline & Control & Nonparetic & Paretic & Control versus nonparetic & Control versus paretic & Nonparetic versus paretic \\
\hline Ankle dorsiflexion angle $\left(^{\circ}\right)$ & $5.3 \pm 1.3$ & $3.3 \pm 1.0$ & $-3.7 \pm 2.8$ & & 0.026 & 0.053 \\
\hline Knee flexion angle $\left(^{\circ}\right)$ & $20.8 \pm 1.8$ & $18.5 \pm 1.3$ & $5.9 \pm 5.5$ & & & \\
\hline Hip flexion angle $\left(^{\circ}\right)$ & $25.6 \pm 3.2$ & $32.2 \pm 2.0$ & $20.8 \pm 3.6$ & & & 0.012 \\
\hline Hip adduction angle $\left(^{\circ}\right)$ & $6.2 \pm 2.1$ & $1.1 \pm 1.3$ & $2.0 \pm 1.7$ & 0.012 & & \\
\hline
\end{tabular}

Values are intragroup mean \pm SE of the mean. Only $p$ values that show significant differences between support leg conditions $(p<0.05)$ have been presented. Stance leg joint angles were calculated at trip onset

models, alongside support leg condition (control, nonparetic, and paretic). Each variable was first separately included in the model along with support leg condition. We examined: (1) whether the variable in question had a significant effect $(p<0.05)$ on onset latencies, and (2) whether with the inclusion of the covariate, limb condition remained a significant effect. If a covariate did not have a significant effect, we concluded that it did not contribute to differences in onset latencies, and it was excluded from subsequent analyses. If support leg condition remained a significant effect after including other covariates, we concluded that the difference between onset latencies across limb conditions could not be accounted for by these confounding effects.

\section{Walking speed}

Across all support leg conditions and all muscles, when walking speed was included as a covariate alongside support leg condition in a repeated-measures mixed-effect analysis of variance, walking speed did not have a significant effect ( $p=0.54$ ) on onset latency, while support leg condition (paretic vs. control, and nonparetic vs. control) remained significant $(p<0.001)$. Walking speed was therefore excluded from the remaining analyses.

\section{Perturbed ankle stiffness}

Perturbed ankle stiffness was estimated using the deviation of the sagittal plane ankle trajectory from unperturbed conditions within $10 \mathrm{~ms}$ of trip onset and an estimate of the trip force moment about the ankle. The force vector direction was along the trip cable. An estimate of the trip cable line of action was obtained using the origin of the cable on the device and a marker on the back of the heel, where the cable was attached. The moment arm of the trip force about the ankle, $l$, was calculated as the vertical distance between the ankle joint center and the cable line of action. The moment of the trip force was $T_{\text {trip }}=l \cdot F_{\text {trip }}$, where $F_{\text {trip }}$ was the measured trip force. Ankle stiffness, $K_{\text {ankle }}$, was calculated as follows: $K_{\text {ankle }}=\mathrm{d} T_{\text {trip }} / \mathrm{d} D_{\text {ankle, }}$, where $\mathrm{d} T_{\text {trip }}=T_{\text {trip }}($ trip onset $+10 \mathrm{~ms})-T_{\text {trip }}($ trip onset $), \quad D_{\text {ankle }}$ was the deviation in ankle trajectory calculated as the perturbed ankle trajectory less the average unperturbed trajectory, and $\mathrm{d} D_{\text {ankle }}=D_{\text {ankle }}($ trip onset $+10 \mathrm{~ms})-D_{\text {ankle }}$ (trip onset $)$.

Estimated values of ankle stiffness are provided in Table 2. These values were within the reported range of values measured for intrinsic stiffness for control, nonparetic, and paretic ankles (e.g., Thajchayapong et al. 2006; Galiana et al. 2005). Across all support leg condition and all muscles, when ankle stiffness and support leg condition were included, ankle stiffness was not a significant effect $(p=0.16)$ on onset latencies, while support leg condition remained significant. Ankle stiffness was therefore excluded from the remaining analysis.

\section{Support leg postures}

Across all support leg conditions and all muscles, when support leg postures (hip, knee, and ankle angles at trip onset) and support leg condition (control, nonparetic, and paretic) were included in the mixed-effect model, support leg postures were not a significant effect $(p>0.30)$, while support leg condition remained significant. Support leg postures at trip onset are presented in Table 3.

\section{References}

Cronin NJ, Ishikawa M, Grey MJ, af Klint R, Komi PV, Avela J, Sinkjaer T, Voigt M (2009) Mechanical and neural stretch responses of the human soleus muscle at different walking speeds. J Physiol 587:3375-3382. doi:10.1113/jphysiol.2008.162610

d'Avella A, Bizzi E (2005) Shared and specific muscle synergies in natural motor behaviors. Proc Natl Acad Sci USA 102:30763081. doi:10.1073/pnas.0500199102

Dietz V (1992) Human neuronal control of automatic functional movements: interaction between central programs and afferent input. Physiol Rev 72:33-69

Dietz V, Berger W (1984) Interlimb coordination of posture in patients with spastic paresis. Impaired function of spinal reflexes. Brain 107(Pt 3):965-978

Eng JJ, Winter DA, Patla AE (1994) Strategies for recovery from a trip in early and late swing during human walking. Exp Brain Res 102:339-349 
Finley JM, Perreault EJ, Dhaher YY (2008) Stretch reflex coupling between the hip and knee: implications for impaired gait following stroke. Exp Brain Res 188:529-540

Finley JM, Dhaher YY, Perreault EJ (2012) Contributions of feed-forward and feedback strategies at the human ankle during control of unstable loads. Exp Brain Res 217:53-66

Finley JM, Dhaher YY, Perreault EJ (2013) Acceleration dependence and task specific modulation of short and medium latency reflexes in the ankle extensors. Physiological reports 1:e00051

Galiana L, Fung J, Kearney R (2005) Identification of intrinsic and reflex ankle stiffness components in stroke patients. Exp Brain Res 165:422-434

Grey MJ, Ladouceur M, Andersen JB, Nielsen JB, Sinkjær T (2001) Group II muscle afferents probably contribute to the medium latency soleus stretch reflex during walking in humans. J Physiol 534:925-933

Haridas C, Zehr EP, Misiaszek JE (2005) Postural uncertainty leads to dynamic control of cutaneous reflexes from the foot during human walking. Brain Res 1062:48-62. doi:10.1016/j. brainres.2005.09.003

Hyndman D, Ashburn A, Stack E (2002) Fall events among people with stroke living in the community: circumstances of falls and characteristics of fallers. Arch Phys Med Rehabil 83:165-170

Kimura T, Nozaki D, Nakazawa K, Akai M, Ohtsuki T (2003) Gradual increment/decrement of isometric force modulates soleus stretch reflex response in humans. Neurosci Lett 347:25-28

Kirker SGB, Simpson DS, Jenner JR, Wing AM (2000) Stepping before standing: hip muscle function in stepping and standing balance after stroke. J Neurol Neurosurg Psychiatry 68:458-464. doi:10.1136/jnnp.68.4.458

Krasovsky T, Lamontagne A, Feldman AG, Levin MF (2013) Reduced gait stability in high-functioning poststroke individuals. J Neurophysiol 109:77-88. doi:10.1152/jn.00552.2012

Littell R, Henry P, Ammerman C (1998) Statistical analysis of repeated measures data using SAS procedures. J Anim Sci 76:1216-1231

Marigold DS, Eng JJ (2006) Altered timing of postural reflexes contributes to falling in persons with chronic stroke. Exp Brain Res 171:459-468

Marigold DS, Eng JJ, Dawson AS, Inglis JT, Harris JE, Gylfadottir S (2005) Exercise leads to faster postural reflexes, improved balance and mobility, and fewer falls in older persons with chronic stroke. J Am Geriatr Soc 53:416-423. doi:10.1111/j.1532-5415.2005.53158.x

Nielsen J, Petersen N, Fedirchuk B (1997) Evidence suggesting a transcortical pathway from cutaneous foot afferents to tibialis anterior motoneurones in man. J Physiol 501:473-484

Ogiso K, McBride J, Finni T, Komi P (2002) Short-latency stretch reflex modulation in response to varying soleus muscle activities. J Electromyogr Kinesiol 12:17-26

Pijnappels M, Bobbert MF, van Dieën JH (2004) Contribution of the support limb in control of angular momentum after tripping. $\mathbf{J}$ Biomech 37:1811-1818

Pijnappels M, Bobbert MF, van Dieën JH (2005a) Control of support limb muscles in recovery after tripping in young and older subjects. Exp Brain Res 160:326-333
Pijnappels M, Bobbert MF, van Dieën JH (2005b) How early reactions in the support limb contribute to balance recovery after tripping. J Biomech 38:627-634

Schillings A, Van Wezel B, Mulder T, Duysens J (2000) Muscular responses and movement strategies during stumbling over obstacles. J Neurophysiol 83:2093-2102

Schmit BD, McKenna-Cole A, Rymer WZ (2000) Flexor reflexes in chronic spinal cord injury triggered by imposed ankle rotation. Muscle Nerve 23:793-803

Shirota C, Simon AM, Kuiken TA (2014) Trip recovery strategies following perturbations of variable duration. J Biomech 47:26792684. doi:10.1016/j.jbiomech.2014.05.009

Sinkjaer T, Andersen JB, Larsen B (1996) Soleus stretch reflex modulation during gait in humans. J Neurophysiol 76:1112-1120

Sinkjaer T, Andersen JB, Nielsen JF, Hansen HJ (1999) Soleus longlatency stretch reflexes during walking in healthy and spastic humans. Clin Neurophysiol 110:951-959

Solnik S, Rider P, Steinweg K, DeVita P, Hortobágyi T (2010) TeagerKaiser energy operator signal conditioning improves EMG onset detection. Eur J Appl Physiol 110:489-498

Stapley PJ, Drew T (2009) The pontomedullary reticular formation contributes to the compensatory postural responses observed following removal of the support surface in the standing cat. J Neurophysiol 101:1334-1350. doi:10.1152/jn.91013.2008

Stevenson AJT, Geertsen SS, Andersen JB, Sinkjær T, Nielsen JB, Mrachacz-Kersting N (2013) Interlimb communication to the knee flexors during walking in humans. J Physiol 591:49214935. doi:10.1113/jphysiol.2013.257949

Stubbs PW, Nielsen JF, Sinkjær T, Mrachacz-Kersting N (2012) Short-latency crossed spinal responses are impaired differently in sub-acute and chronic stroke patients. Clin Neurophysiol 123:541-549

Swinnen SP, Serrien DJ, Walter CB, Philippaerts R (1995) The organization of patterns of multilimb coordination as revealed through reaction time measures. Exp Brain Res 104:153-162

Thajchayapong M, Alibiglou L, Lilaonitkul T, Mirbagheri M (2006) Mechanical abnormalities of the spastic ankle in chronic stroke subjects. Conf Proc IEEE Eng Med Biol Soc 1:3688-3691

Toft E, Sinkjær T, Andreassen S (1989) Mechanical and electromyographic responses to stretch of the human anterior tibial muscle at different levels of contraction. Exp Brain Res 74:213-219

Toft E, Sinkjaer T, Andreassen S, Larsen K (1991) Mechanical and electromyographic responses to stretch of the human ankle extensors. J Neurophysiol 65:1402-1410

van Swigchem R, van Duijnhoven HJ, den Boer J, Geurts AC, Weerdesteyn V (2013) Deficits in motor response to avoid sudden obstacles during gait in functional walkers poststroke. Neurorehabil Neural Repair 27:230-239. doi:10.1177/1545968312462070

Weerdesteyn V, de Niet M, van Duijnhoven HJR, Geurts ACH (2008) Falls in individuals with stroke. J Rehabil Res Dev 33:36

Zehr EP, Loadman PM (2012) Persistence of locomotor-related interlimb reflex networks during walking after stroke. Clin Neurophysiol 123:796-807. doi:10.1016/j.clinph.2011.07.049 